\title{
Análise de desempenho de ovelhas e cordeiros Ile de France
}

\author{
Performance analysis of ewe and lambs Ile de France \\ Análisis del rendimiento de ovejas y corderos Ile de France
}

Recebido: 31/01/2022 | Revisado: 05/02/2022 | Aceito: 11/02/2022 | Publicado: 17/02/2022

Ana Lúcia Barreto da Costa
ORCID: https://orcid.org/0000-0002-0807-2581
E-mail: ad.costa1998@gmail.com
Thaís Campos de Freitas
Instituto Federal de Educação, Ciência e Tecnologia do Ro Grande do Sul, Brasi
ORCID: https://orcid.org/0000-0003-3942-599X
E-mail: thaiscamposdefreitas@ @mail.com
Elísio de Camargo Debortoli
Instituto Federal de Educação, Ciência e Tecnologia Ro Rio Grande do Sul, Brasil
ORCID: https://orcid.org/0000-0001-5594-2810
E-mail: elisio.debortoli @ sertao.ifrs.edu.br
Edriane Mara Sbardelotto
Instituto Federal de Educação, Ciência e Tecnologia do Rio Grande do Sul, Brasil
ORCID: https://orcid.org/0000-0003-2985-5996
Instituto Federal de Educação, Ciência e Tecnologia do Rio Grande do Sul, Brasil
E-mail: edrianesbardelotto@gmail.com
Jaqueline Schneider Lemes
ORCID: https://orcid.org/0000-0001-7173-8107
Universidade Federal de Santa Maria, Brasil
E-mail: jaqueline.lemes@ @ufsm.br

\section{Resumo}

Os sistemas de produção dos ovinos no Rio Grande do Sul apresentam-se de forma diversificada. Como consequência destes sistemas adotados, alguns índices produtivos e reprodutivos médios do rebanho tornam-se inferiores ao potencial dos rebanhos. O objetivo deste estudo foi avaliar o desempenho produtivo de ovelhas e cordeiros da raça Ile de France criados em sistema extensivo em campo nativo na mesorregião Nordeste do Rio Grande do Sul. O experimento foi realizado na Cabanha Lagoa, localizada no município de Capão Bonito do Sul. Foram utilizados dados de manejos de 33 matrizes ovinas e 37 cordeiros, da raça Ile de France. Os dados referem-se aos ciclos de produção de cordeiros nascidos em 2018, acompanhados até os 340 dias de vida e desempenho de matrizes do ciclo de produção de 2019. Ao avaliar a eficiência das matrizes, levando em consideração o sexo dos cordeiros, constatouse que não houve diferença significativa tanto para o peso ao parto quanto ao desmame. Para a eficiência das matrizes de acordo com o tipo de parto (simples ou múltiplo), foi possível identificar que a eficiência da ovelha ao parto (EFp) foi superior nos partos múltiplos. Já os cordeiros de partos simples obtiveram maior peso em $\mathrm{Kg}$ que os cordeiros de parto múltiplo. Matrizes de partos múltiplos apresentaram desempenho superior (104\% de seu peso vivo desmanado) em relação à eficiência produtiva se comparados com matrizes de parto simples (65\% de seu peso desmamado). Em relação à evolução de peso, cordeiros de parto simples obtiveram pesos superiores do que animais de partos múltiplos. Palavras-chave: Eficiência produtiva; Tipo de parto; Ovinos; Matrizes.

\begin{abstract}
Sheep production systems in Rio Grande do Sul are diversified. Thus, some reproductive and productive indices of the herd become smaller than the potential of the herds. The aim of this study was to evaluate the productive performance of Ile de France ewes and lambs reared extensively in a native field located in the Northeast mesoregion of Rio Grande do Sul. The experiment was developed in Cabanha Lagoa, located in the municipality of Capão Bonito do Sul. Data from of 33 Ile de France sheep and 37 lambs. The data refer to the production cycles of lambs born in 2018 and performance of sows from the 2019 production cycle followed up to 340 days of life. When evaluating the efficiency of the dams, taking into account the sex of the lambs, it was found that there was no significant difference for both birth and weaning weight. For the efficiency of the dams according to the type of birth (single-born or multiple-born), it was possible to identify that the efficiency of the ewe at birth (Efp) was higher in multiple-born. Lambs from single-born, on the other hand, had greater weight in $\mathrm{kg}$ than lambs from multiple-born. Dams from multiple-born presented superior performance (104\% of their weaned weight) in relation to productive efficiency when compared to dams from single-born (65\% of their weaned weight). With regard to weight evolution, lambs from single-born obtained higher weights in a shorter time, compared to lambs from multiple-born.
\end{abstract}

Keywords: Dams; Sheep; Productive efficiency; Type of born. 


\begin{abstract}
Resumen
Los sistemas de producción de ovinos en Rio Grande do Sul están diversificados. Como consecuencia de estos sistemas adoptados, algunos índices productivos y reproductivos medios se vuelven menor que el potencial de los rebaños. El objetivo de este estudio fue evaluar el desempeño productivo de ovejas y corderos de la raza Ile de France criados en sistema extensivo en un campo nativo en la mesorregión nororiental de Rio Grande do Sul. El experimento fue realizado en Cabanha Lagoa, ubicada en el municipio de Capão Bonito do Sul se utilizaron datos de manejo de 33 ovejas y 37 corderos de la raza Ile de France. Los datos se refieren a los ciclos de producción de los corderos nacidos en 2018, seguidos hasta los 340 días de vida y desempeño de las matrices del ciclo de producción de 2019. Al evaluar la eficiencia de las matrices, teniendo en cuenta el sexo de los corderos, se encontró Se encontró que no hubo diferencia significativa tanto para el peso al nacer como para el peso al destete. Para la eficiencia de las cerdas según el tipo de parto (único o múltiple), se pudo identificar que la eficiencia de la oveja al parto (Efp) fue mayor en los partos múltiples. Por otro lado, los corderos de parto único obtuvieron mayor peso en $\mathrm{kg}$ que los corderos de parto múltiple. Las cerdas de parto múltiple mostraron un rendimiento superior (104\% de su peso vivo destetado) en términos de eficiencia productiva en comparación con las cerdas de parto único (65\% de su peso destetado). En cuanto a la evolución del peso, los corderos de parto único obtuvieron pesos superiores a los de parto múltiple.
\end{abstract}

Palabras clave: Eficiencia productiva; Tipo de parto; Oveja; Matrices.

\title{
1. Introdução
}

No Rio Grande do Sul a ovinocultura caracteriza-se pela criação de forma extensiva, onde os animais são manejados exclusivamente em pastagem natural (campo nativo), levando em consideração os aspectos produtivos, econômicos e de sustentabilidade os quais possuem relevância para que o produtor permaneça na atividade. No entanto, estratégias de terminação, principalmente em confinamento, vêm ganhando destaque próximo a centros consumidores, devido a um aumento significativo no rebanho ovino, onde no ano de 2020 o rebanho ovino no Brasil apresentou um crescimento de 3,3\% em relação ao efetivo registrado em 2019, totalizando 20.628.699 cabeças aumento esse que se deve tanto ao interesse econômico quanto ao mercado consumidor (EMBRAPA, 2021).

No passado, a produção era voltada para consumo familiar, mas com o passar do tempo foi possível atingir outras finalidades, observando que esta atividade gerava um novo cenário econômico. A ovinocultura de corte é uma das atividades com maior potencial de crescimento, dentre as atividades pecuárias no Brasil (Barreto Neto, 2010; Geron et al., 2012). Porém a carne ovina ainda tem produção e consumo tímidos, o que faz com que esse produto tenha grande potencial de crescimento (SEBRAE, 2020).

A raça Ile de France, especializada na produção de carne, é de origem francesa e foi introduzida no Brasil no início da década de 1970. Adaptou-se bem às condições climáticas, quanto à fertilidade e a velocidade de crescimento dos cordeiros, principalmente, quando utilizada como raça paterna em ovelhas lanadas ou de duplo propósito. Das raças especializadas na produção de carne, a Ile de France é a que tem melhor lã, além de apresentar menor estacionalidade reprodutiva, sendo pouco afetada pelo fotoperíodo, quando comparadas as outras raças ovinas de origem europeia (ABCIF, 2021).

Um dos empasses para o progresso da ovinocultura no Brasil é o baixo desempenho produtivo, oriundo de manejos inadequados. O desempenho dos rebanhos pode ser mensurado através de quilogramas de cordeiros desmamados por ovelha, sendo influenciada por fatores ambientais, individuais e genéticos (Ribeiro et al., 2013). Destacando também a importância do uso da seleção para a avaliação deste rebanho. Sendo possível através dela, identificar e selecionar animais superiores geneticamente permitindo assim a permanência no rebanho apenas de animais que tenham gerado cordeiros.

O desempenho dos animais pode ser refletido através de diversos fatores, estes podem ser inerentes aos animais, tais como: sexo do cordeiro, tipo de nascimento (simples ou gemelar), época de nascimento, efeitos maternais e idade das ovelhas (Aita, 2010). Em virtude da crescente demanda na produção de carne ovina, surge a necessidade de avaliarmos os animais quanto à eficiência produtiva, que pode ser afetada pela combinação entre fatores ambientais e genéticos.

Para que a atividade de ovinocultura possa apresentar resultados favoráveis ou expressivos, se faz necessário, antes de tudo, um controle zootécnico que possibilite ao produtor identificar animais superiores geneticamente, sendo assim podemos 
utilizar alguns critérios de seleção, para machos, saber sua origem, avaliar aprumos, testículos se estão em tamanhos adequados sem nenhuma anomalia, além de outras características como efeitos hereditários (prognatismo). Para fêmeas podem-se observar características como, aspecto feminino, bom desenvolvimento corporal, cascos sadios, números de partos etc. E deste modo pode-se dar sequência ao programa de melhoramento, selecionando animais com maior capacidade produtiva e reprodutiva. Sendo assim, para superar estas barreiras, se faz necessário avaliar seus potenciais de produção determinando quais são mais produtivos, em quais categorias de produção se encaixam, (ovinos leiteiros e ovinos de corte) e se devem ou não receber tratamento diferenciado para uma maior uniformidade de produção.

Segundo Oliveira et al. (2010), uma maneira mais consistente de analisar a eficiência produtiva de ovinos, seria através do estudo de curvas de crescimento. Essas curvas relacionam o peso do animal, com a idade, e utilizam modelos não lineares para sintetizar grande volume de informações em um pequeno conjunto de parâmetros que podem ser interpretados biologicamente (Teixeira et al., 2011). Importante salientar a eficácia da coleta de dados, ela requer tempo e fidelidade nas anotações. Muitas vezes o produtor acaba por não fazer as anotações, prejudicando assim o processo de seleção dos rebanhos. Tanto a coleta, como as análises realizadas são importantes parar gerar índices zootécnicos precisos e confiáveis.

Desta maneira, o objetivo deste estudo foi avaliar o desempenho produtivo de ovelhas e cordeiros da raça Ile de France em sistema extensivo em campo nativo.

\section{Metodologia}

O experimento foi realizado na Cabanha Lagoa, que está localizada no munícipo de Capão Bonito do Sul, Rio Grande do Sul, latitude: $28^{\circ}$ 6' 6" Sul, longitude: 51 23' 24" Oeste. A região de Capão Bonito do Sul apresenta um clima subtropical úmido. Segundo EMBRAPA (2021), a classificação do clima é Cfa com predomínio de temperaturas superiores a $22{ }^{\circ} \mathrm{C}$ no verão e com mais de $30 \mathrm{~mm}$ de chuva nos meses mais secos. Temperatura moderada, chuvas bem distribuídas, verão quente e apresenta geada nos meses de inverno com temperatura média no inverno abaixo de $16^{\circ} \mathrm{C}$ e temperatura máxima no mês mais quente de $30^{\circ} \mathrm{C}$.

Foram utilizados dados de manejos de 33 matrizes ovinas e 37 cordeiros sendo 18 machos não castrados e 19 fêmeas, da raça Ile de France, criadas em sistema extensivo, a pasto (campo nativo), com fornecimento de água e sal mineral $a d$ libitum. Os dados referem-se ao ciclo de produção de cordeiros nascidos em 2018 e acompanhados até os 340 dias de vida, finalizando em 2019. Já o desempenho das matrizes, refere-se ao ciclo de produção do ano de 2019. Para as matrizes foram utilizadas informações de peso adulto, tipo de parto (simples ou gemelar), sexo do cordeiro, e pesagens, ao nascer, aos trinta dias e peso ao desmame, esse desmame ocorre aos 4 meses. Dessa maneira criou-se os indicadores: Eficiência das ovelhas ao parto $(E F p)$ e Eficiência da produção de cordeiros ao desmame $(E F c d)$, conforme as equações a seguir:

$$
\begin{gathered}
E F p=\frac{\text { Somatório do peso dos cordeiros ao nascimento }}{\text { Somatório do peso das ovelhas ao encarneiramento }} \times 100 \\
E F c d=\frac{\text { Somatório do peso dos cordeiros ao desmame }}{\text { Somatório do peso das ovelhas ao encarneiramento }} \times 100
\end{gathered}
$$

Já para os cordeiros foram considerados tipo de parto oriundo (simples ou gemelar), sexo do cordeiro e suas respectivas pesagens, sendo consideradas dez pesagens: Pnasc (peso ao nascimento), P1 (peso aos 30 dias), P2 (peso aos 120 dias), P3 (peso aos 160 dias), P4 (peso aos 190 dias), P5 (peso aos 220 dias), P6 (peso aos 250 dias), P7 (peso aos 280 dias), 
P8 (peso aos 310 dias) e, P9 (peso aos 340 dias). Os dados foram submetidos a análise de variância (ANOVA) considerando diferença sifnificativa de 5\%, sendo a comparação das médias realizada pelo Teste de Tukey a 5\% de significância.

\section{Resultados e Discussão}

A Tabela 01 apresenta os resultados em relação à avaliação de EFp e da EFcd. Foi possivel identificar, avaliando o sexo dos cordeiros, que não houve diferença significativa $(\mathrm{P}<0,05)$, tanto para o peso ao parto quanto para peso ao desmame.

O desempenho dos ovinos pode variar com diversos parâmetros tais como: sexo, pesagem, nutrição e também o tipo de nascimento, onde é observado que animais oriundos de parto simples apresentam um ganho de peso diário superior aos nascidos de parto gemelar. Essa diferença está atrelada ao ambiente intrauterino e a capacidade de produção de leite das matrizes, sofrendo influência direta do pré e pós-parto devido ao seu estado nutricional.

Na Tabela 1, observa-se a média e o desvio padrão dos cordeiros, para os indicadores de eficiência, de acordo com o sexo.

Tabela 1 - Médias e Desvio Padrão para eficiência das matrizes por sexo do cordeiro produzido.

\begin{tabular}{ccc}
\hline Sexo & EFp & EFcd \\
& (MED e SD) & $48,89 \pm 13,83$ \\
Fêmeas & $4,69 \pm 1,39$ & $55,40 \pm 14,09$ \\
Machos & $5,24 \pm 0,99$ & NS \\
Significância & NS & .
\end{tabular}

Fonte: Dados da pesquisa.

Fernandes et al. (2001 a), ao trabalharem com rebanho da raça Morada Nova, e comparando os pesos nos diferentes sexos, encontraram pesos superiores nos cordeiros machos tanto ao nascimento quanto ao desmame, pois é de conhecimento que o desenvolvimento de machos é superior desde a gestação. No presente estudo para variavel sexo, não houve diferença significativa. Mas ao obtermos estes resultados apresentados na Tabela 1, podemos chegar a algumas hipóteses que nos levam a entender o porque esses animais não apresentaram diferença significativa na eficiência produtiva em ambas as situações avaliadas para o sexo.

O fato de que a maior parte dos animais do presente estudo foi oriunda de partos múltiplos pode, em partes, justificar o resultado encontrado, pois ovelhas de parto múltiplo produzem cordeiros mais leves, uma vez que estes sofrem por competição no aleitamento e por espaço intrauterino. Entretanto este fato pode não necessariamente ser devido apenas ao tipo de parto visto que estes animais se recuperam bem posteriormente, mas também devido ao aporte nutricional que estão submetidos. Assim como o tipo de parto pode ter interferido no desempenho dos cordeiros, o aporte nutricional ofertado tem grande interferência no desempenho tanto da matriz quanto dos cordeiros.

Carneiro et al. (2004) justificam que, mesmo que as matrizes de parto duplo produzissem mais leite que as de parto simples, a quantidade de leite disponível a cada cordeiro de parto múltiplo é inferior quando comparando com o volume de leite disponível aos cordeiros de parto simples. É de conhecimento a existência de melhor desempenho em animais advindos de partos simples, uma vez que o leite produzido pela matriz não irá ser dividido com mais cordeiros, obtendo uma taxa de crescimento maior, ao contrário de animais advindos de partos múltiplos.

Animais oriundos de partos simples são mais pesados do que aqueles de parto gemelar, o tipo de parto (simples ou duplo) pode influenciar no peso ao nascer e nas demais idades, mas isso não justifica que animais de parto múltiplo não se recuperam bem (Rech et al, 2008). 
No presente estudo não foi possível observar diferença em relação ao peso dos cordeiros e ao sexo dos mesmos. Sendo destas 19 fêmeas, destas 7 de partos simples e 12 de parto múltiplo e 14 machos e destes 10 animais de parto múltiplo e 4 animais de parto simples

Existem diversos fatores que podem influenciar o crescimento fetal de cordeiros, como nível de nutrição materna, sendo de extrema importância, pois irá refletir no desempenho do cordeiro, além do sexo do feto e do tipo de parto, dentre outros aspectos. É importante ressaltar a influência que o nível nutricional que a matriz esta recebendo, principalmente no terço final da gestação, onde ocorre maior desenvolvimento fetal, desta forma, interferindo de forma direta o desempenho do cordeiro. Para Ferrel (1992), uma vez que as matrizes sofrem restrição alimentar, principalmente durante o terço final da gestação, esta irá afetar diretamente no peso dos cordeiros.

Avaliando a relação de quilos desmamados por ovelha, na Tabela 02, foi possível verificar que a eficiência da ovelha ao parto (EFp) foi superior nos partos múltiplos devido a soma dos pesos dos cordeiros, o que não ocorre em animais de partos simples. Já os cordeiros ao desmame de partos simples obtiveram maior peso, em $\mathrm{Kg}$, quando comparados aos de parto múltiplo. Assim, confirmou-se que os animais oriundos de partos simples pesam mais que os desmamados de partos múltiplos.

Na Tabela 2, observa-se o resultado de EFp e EFcd para as matrizes, de acordo com o tipo de parto do cordeiro, sendo somado o peso dos cordeiros de partos múltiplos.

Tabela 2 - Médias e Desvio-Padrão para eficiência das matrizes por tipo de parto, sendo somado o peso dos cordeiros de partos múltiplos.

\begin{tabular}{ccc}
\hline Tipo de parto & $\begin{array}{c}\text { EFp } \\
\text { (MED e SD) }\end{array}$ & $\begin{array}{c}\text { EFcd } \\
\text { (MED e SD) }\end{array}$ \\
\hline Simples & $5,70 \pm 1,92$ & $67,16 \pm 17,43$ \\
Múltiplo & $11,12 \pm 2,46$ & $107,77 \pm 27,71$ \\
Significância & $\mathrm{S}(\mathrm{p}=0,004)$ & $\mathrm{S}(\mathrm{p}=0,0033)$ \\
\hline
\end{tabular}

Fonte: Dados da pesquisa.

Cordeiros nascidos de partos simples apresentam desempenho superior se comparado aos nascimentos duplos, segundo Silva e Araújo (2000), este fato se deve em função da inexistência de competição pelo leite materno. O desempenho produtivo de cordeiros advindos de parto gemelar é considerado inferior ao dos nascidos de partos simples, devido à baixa ingestão de leite materno (Muniz et al., 1997; Carneiro et al., 2004). No entanto, quando comparamos a eficiência produtiva por animal ou por área, para produção de carne, a presença de partos duplos reflete na maior produtividade do sistema como um todo.

Outro fato que explica os pesos superiores de cordeiros de parto simples em relação aos gemelares está relacionado ao ambiente intrauterino e a capacidade de produção de leite das matrizes. O espaço físico uterino torna-se limitado ao crescimento, quando o número de fetos presentes no útero aumenta, o que pode influenciar o peso ao nascer dos cordeiros e seu crescimento final, que pode ser explicado pela correlação negativa entre prolificidade e peso ao nascer (Rêgo Neto et al., 2014).

Machado et al. (1999), estudando cruzamentos entre diferentes raças, relataram que crias de nascimento simples foram mais pesadas quando comparadas as de nascimento duplos, resultado semelhante ao encontrado neste estudo, porém se obtivermos uma boa nutrição, os índices apresentados tendem a melhorar. Para Ribeiro et al. (2002), quando falamos em pesos individuais os cordeiros gêmeos apresentam peso individual menor e, consequentemente, apresentam maior mortalidade até o 
desmame, sendo vantajoso apenas maior número de partos gemelares, se os cordeiros obtiverem um bom peso ao nascimento e se a matriz possui habilidade materna, para reduzir a mortalidade e melhorar os pesos a desmama.

Fernandes et al. (2001 b), analisando registros de um rebanho de ovinos da raça Morada Nova, observaram que o tipo de parto foi importante fonte de variação para explicar as diferenças nos pesos corporais ao nascimento, ao desmame, aos seis meses e aos 12 meses de idade. Sendo que cordeiros nascidos de parto simples pesaram mais que os nascidos de partos duplos.

No presente trabalho as matrizes de parto múltiplo apresentaram desempenho superior na eficiência de produtividade como podemos observar na Tabela 3, onde os resultados apontam que essas matrizes desmamaram valor superior ao seu peso corporal, o que difere dos demais estudos encontrados em que as matrizes geralmente desmamam o seu peso corporal, se mostrando mais eficiente que as de parto simples que desmamaram cerca de $65 \%$ de seu peso corporal em $\mathrm{kg}$ de cordeiro.

Neste estudo, a eficiência produtiva das matrizes de parto múltiplo pode ser explicada pela alimentação que recebem nos piquetes de pastagem, pois ficam alocadas em piquetes que em uma parte do ano tem acesso a áreas agrícolas com pastagem de aveia, azevém e trevo branco. Porém essa eficiência pode ser estar atrelada devido ao fato de ambos, tanto ovelha quanto cordeiro permanecerem nessa pastagem.

Na Tabela 3, observa-se o resultado de EFp e EFcd para as matrizes, de acordo com o tipo de parto do cordeiro, sendo somado o peso dos cordeiros de partos múltiplos.

Tabela 3 - Índices para eficiência das matrizes por tipo de parto.

\begin{tabular}{cccc}
\hline Índice & $\begin{array}{c}\text { Matrizes } \\
\text { parto simples }\end{array}$ & $\begin{array}{c}\text { Matrizes } \\
\text { parto múltiplo }\end{array}$ & P \\
\hline $\mathrm{EFp}$ & 5,54 & 11,10 & $<0,01$ \\
$\mathrm{EFcd}$ & 65,53 & 104,09 & $<0,01$ \\
\hline
\end{tabular}

Fonte: Dados da pesquisa.

Matrizes que criam cordeiros resultantes de partos múltiplos proporcionam mais unidades de abate, sendo um ponto positivo para o mercado de ovinos de corte, cada vez mais crescente. Para Carneiro et al. (2004), a obtenção de partos duplos permite maior eficiência na produção de carne ovina como já mencionado, embora esses animais atinjam peso para abate mais tardio que os animais de partos simples.

De acordo com Manzoni et al. (2017), a eficiência produtiva das ovelhas é indicada através da quantidade de quilogramas produzidos para cada $100 \mathrm{Kg}$ de matriz, sendo calculada no parto e no desmame. É importante mencionar também que as ovelhas que apresentam boa condição corporal no parto, produzem mais leite e consequentemente conseguem desmamar animais mais pesados (Castro et al., 2012). Outro fato que pode justificar a melhor eficiência de matrizes de parto múltiplo, no presente trabalho, pode estar relacionado com o ECC que se manteve adequado durante o período.

Segundo Barros et al. (2005), o ECC da matriz é fundamental para o desenvolvimento do cordeiro na fase de aleitamento, pois será nesta fase que o cordeiro tem maior velocidade de crescimento em ganho de peso, quando seu alimento principal é o leite. Ressaltando que não apenas na fase de aleitamento o escore é fundamental, mas nas demais fases produtivas é de suma importância para a maior produtividade e eficiência.

Além dos conhecimentos de fatores que alteram a eficiência dos cordeiros, a determinação da idade da matriz é primordial para avaliar o desenvolvimento e evolução dos animais, preconizando categorias que tenham seus desenvolvimentos prejudicados, objetivando interação entre genótipo e ambiente, tornando a produção mais homogênea e rentável para o produtor (Ribeiro et al., 2013).

Na Tabela 04, é possível observar a evolução dos pesos dos cordeiros desde o nascimento até os 340 dias de idade, onde foi constatado que os animais oriundos de partos simples obtiveram os maiores pesos tanto ao nascer quanto nas demais 
pesagens. Ao todo foram nove pesagens que mostram a eficiência em ganho de peso dos animais provenientes de partos simples. As diferenças identificadas entre os dois tipos de partos foram significativas.

$\mathrm{Na}$ Tabela 4, observa-se a evolução de peso dos cordeiros de parto simples e múltiplos do nascimento aos 340 dias de idade.

Tabela 4 - Média e Desvio-Padrão para o peso dos cordeiros do nascimento aos 340 dias de idade por tipo de parto.

\begin{tabular}{|c|c|c|c|}
\hline Peso & $\begin{array}{l}\text { Parto simples } \\
\qquad(\mathrm{N}=5)\end{array}$ & $\begin{array}{l}\text { Parto múltiplo } \\
\quad(\mathbf{N}=32)\end{array}$ & Valor de $P$ \\
\hline Pnasc & $4,66 \pm 0,64$ & $3,74 \pm 0,70$ & $<0,001 * *$ \\
\hline $\mathrm{P} 1$ & $12,84 \pm 1,63$ & $8,11 \pm 1,68$ & $<0,0001 * * *$ \\
\hline $\mathrm{P} 2$ & $50,20 \pm 9,65$ & $37,56 \pm 6,89$ & $<0,0001 * * *$ \\
\hline P3 & $55,0 \pm 8,77$ & $41,72 \pm 5,84$ & $<0,0001 * * *$ \\
\hline P4 & $62,76 \pm 7,80$ & $48,80 \pm 5,62$ & $<0,0001 * * *$ \\
\hline P5 & $62,72 \pm 8,95$ & $48,39 \pm 6,11$ & $<0,0001 * * *$ \\
\hline P6 & $66,28 \pm 8,70$ & $51,77 \pm 6,95$ & $<0,0001 * * *$ \\
\hline P7 & $69,20 \pm 4,39$ & $57,47 \pm 6,95$ & $<0,0001 * * *$ \\
\hline P8 & $72,12 \pm 5,50$ & $59,33 \pm 7,25$ & $<0,0001 * * *$ \\
\hline P9 & $78,68 \pm 6,55$ & $62,06 \pm 7,37$ & $<0,0001 * * *$ \\
\hline
\end{tabular}

Nota: Pnasc = peso ao nascimento; $\mathrm{P} 1$ = peso aos 30 dias; $\mathrm{P} 2$ = peso aos 120 dias; $\mathrm{P} 3$ = peso aos 160 dias; $\mathrm{P} 4=$ peso aos 190 dias; $\mathrm{P} 5=$ peso aos 220 dias; P6 = peso aos 250 dias; $\mathrm{P} 7$ = peso aos 280 dias; $\mathrm{P} 8=$ peso aos 310 dias e; P9 = peso aos 340 dias. Fonte: Autores.

É possível considerar o sexo e o número de cordeiros nascidos por parto como um dos dois fatores que possuem maior influência no crescimento dos ovinos, onde estes fatores afetam significativamente a rentabilidade dos sistemas intensivos de produção onde se objetiva maior eficiência no menor tempo possível. Para Silva e Araújo (2000) os tipos de nascimentos influenciam significativamente todos os pesos e ganho de peso. Foi possível observar o mesmo no presente trabalho, pois os animais de parto simples apresentaram melhor peso nas nove pesagens comparado aos de parto múltiplo.

No Gráfico 1 pode-se observar o tempo de ganho de peso dos cordeiros, destacando que os animais de parto simples obtiveram ganho de peso superior aos de parto múltiplo. Este fato pode ser explicado pela oferta de alimento (leite) aos cordeiros de parto simples. Estes não precisaram competir/dividir o leite materno com outros cordeiros, no entanto, os cordeiros de parto múltiplo conseguiram atingir o peso para abate, (que na propriedade os animais vão para abate aos $42 \mathrm{Kg}$ ) devido ao aporte nutricional que recebem em pastagem melhorada. 
Gráfico 1 - Evolução do peso dos cordeiros de parto simples e múltiplo.

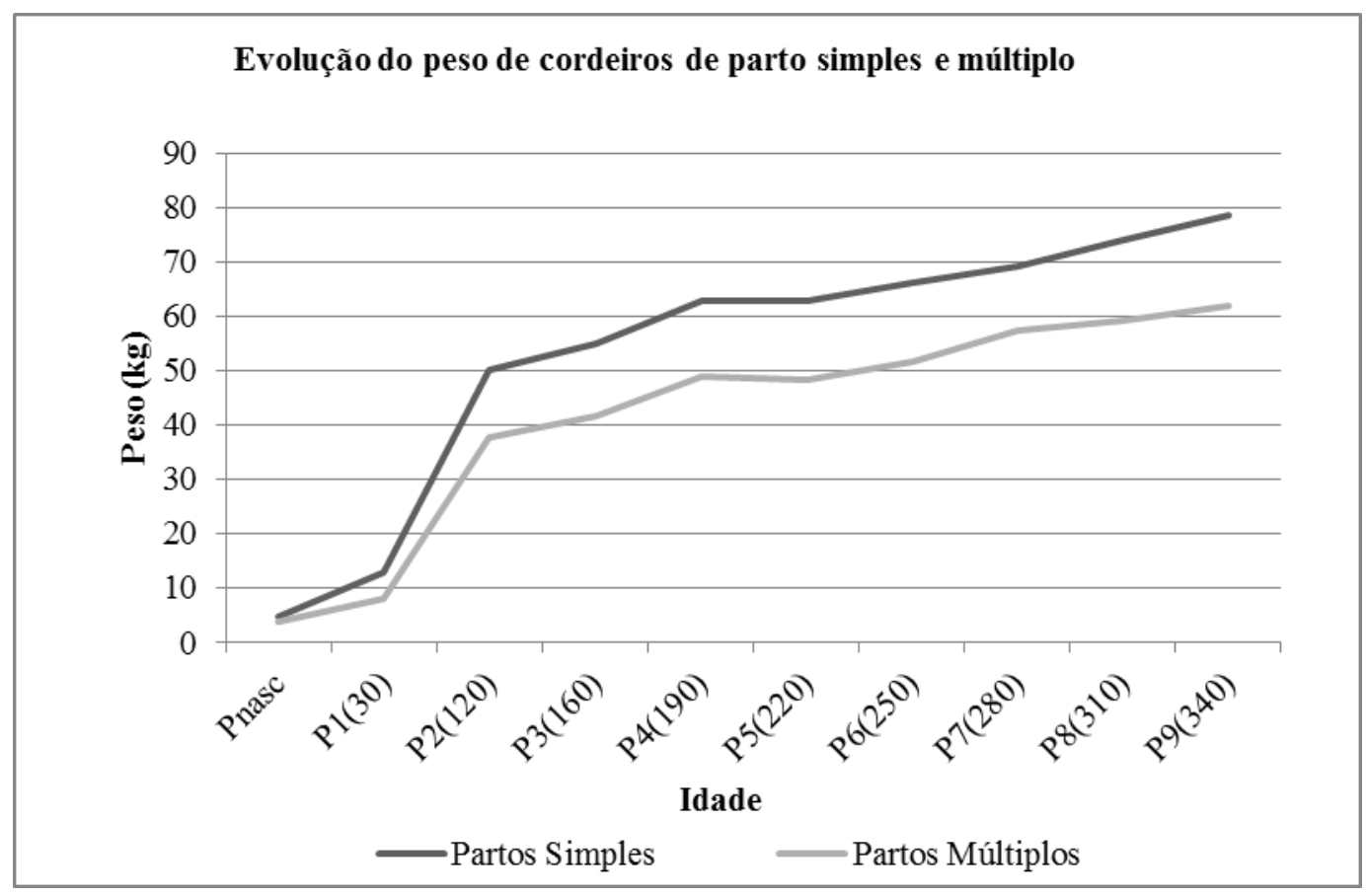

Fonte: Dados da pesquisa.

Carneiro et al. (2004), em estudo realizado no departamento de Zootecnia da UFSM- RS, com o objetivo de avaliar o ganho de peso, consumo de matéria seca e conversão alimentar de cordeiros oriundos de parto simples e duplo, desmamados aos 63 dias e, de parto simples não desmamados, confinados e abatidos aos $30 \mathrm{~kg}$, constataram que nos primeiros 21 dias de lactação o ganho de peso médio diário dos cordeiros oriundos de parto duplo foi inferior aos provenientes de parto simples e que, do nascimento ao abate, cordeiros de parto simples desmamados obtiveram menor ganho de peso que os cordeiros de parto simples não desmamados. Neste período o menor ganho de peso foi dos cordeiros provenientes de parto duplo, ocasionando um maior tempo para atingir o peso de abate (126 dias), enquanto que os cordeiros de parto simples atingiram o peso de abate em menos tempo ( 87 dias).

Um fato que pode explicar a baixa evolução dos cordeiros de parto múltiplo, além da competição por leite materno e menor produção pela matriz, pode estar relacionado com a idade das ovelhas, onde ovelhas mais jovens por estarem em fase de desenvolvimento acabam gerando cordeiros mais leves e estes obtêm menores ganhos de peso e são mais leves no desmame (Ribeiro et al., 2009).

O fato de matrizes produzirem cordeiros de partos múltiplos não é um ponto negativo, muito pelo contrário a obtenção de partos múltiplos vai proporcionar uma maior eficiência na produção de carne ovina e quando comparamos a eficiência produtiva por animal ou por área, para produção de carne, a presença de partos múltiplos reflete na maior produtividade do sistema. Ainda, corroborando com o presente trabalho, Rocha et al. (2009) encontraram que o tipo de nascimento influenciou o desempenho dos cordeiros do nascimento ao desmame, onde nascidos de parto simples foram superiores aos de parto gemelar. Para Mohammadi et al. (2010), a principal razão pelo qual animais de partos gemelares tem menor desenvolvimento no período que corresponde ao pré-desmame é a competição intrauterina, seguida pela competição pelo leite materno.

Fato que pode vir a contribuir para entendermos o desenvolvimento mais lento dos cordeiros gemelares neste estudo, uma vez que sofreram, principalmente, pela competição por leite materno, reduzindo suas taxas de ganho de peso. Além de outros fatores, como o ambiente em que estão inseridos, o aporte nutricional e demais características que podem ter interferido no desempenho dos cordeiros. 


\section{Considerações Finais}

Com este trabalho foi possível concluir que, na avaliação da eficiência das matrizes estudadas, levando em consideração o sexo dos cordeiros, não houve diferença significativa, tanto para o peso ao parto, quanto ao desmame. Para a eficiência das matrizes de acordo com o tipo de parto (simples ou múltiplo), foi possível identificar que a eficiência da ovelha ao parto (Efp), foi superior nos partos múltiplos. Já os cordeiros de partos simples obtiveram maior peso em $\mathrm{Kg}$ que os cordeiros de parto múltiplo.

Matrizes de partos múltiplos apresentaram desempenho superior, desmamando 104\% de seu peso vivo em relação a eficiência produtiva das matrizes de parto simples que desmamaram $65 \%$ de seu peso vivo. No que diz respeito à evolução de peso, cordeiros de parto simples obtiveram pesos superiores em menor tempo, comparados com animais nascidos de partos múltiplos.

\section{Agradecimentos}

Ao proprietário da Cabanha Lagoa pela concessão dos dados de manejo de sua propriedade, fundada em 2003, pelo proprietário e engenheiro agrônomo César A. Magrin, destinada à produção de ovinos das raças Ile de France, Texel e suas cruzas, em sistema semi-intensivo.

\section{Referências}

ABCIF - Associação Brasileira de Criadores de Ile de France. Características (2021). www.iledefrance.org.br/caracteristicas.php.

Alta, M. F (2010). Efeitos do temperamento sobre o comportamento materno de ovelhas e o desenvolvimento corporal de seus cordeiros. 2010. 184f. Tese (Doutorado em Zootecnia) - Faculdade de Agronomia, Universidade Federal do Rio Grande do Sul, Porto Alegre, RS.

Barreto Neto, A. D (2010). Posicionamento estratégico do setor de carnes de caprinos e ovinos no mercado de carnes brasileiro. Revista Tecnologia \& Ciência Agropecuária, 4(4), 81-85.

Barros, N. N., Cavalcante, A. C., \& Bomfim, M. A. D (2005). Alimentação e manejo alimentar. In: Cavalcante, A. C. R, Barros, N. N. Sistema de Produção de Caprinos e Ovinos de Corte para o Nordeste Brasileiro. Brasília: Embrapa Informação Tecnológica, 1, 155-194.

Carneiro, R. M., Pires, C. C, Müller, L., Kippert, C. J., Costa, M. L., Colomé, L. M \& Osmari, E.K (2004) Ganho de peso e eficiência alimentar de cordeiros de parto simples e duplo desmamados aos 63 dias e não desmamados. Revista Brasileira de Agrociência, 10:227-230.

Castro, F. A. B., Ribeiro, E. L. A., \& Koritiaki,, N. A. et al (2012). Desempenho de cordeiros Santa Inês do nascimento ao desmame filhos de ovelhas alimentadas com diferentes níveis de energia. Semina: Ciências Agrarias, 33, 3379-3388.

Empresa Brasileira De Pesquisa Agropecuaria - EMBRAPA (2021). Sistema de classificação climática. https://www.cnpf.embrapa.br/pesquisa/efb/clima.htm.

Empresa Brasileira De Pesquisa Agropecuária- EMBRAPA Caprinos e Ovinos (2021). Boletim No 16 | Sobral, CE, outubro, Pesquisa Pecuária Municipal 2020: rebanhos de caprinos e ovinos. https://ainfo.cnptia.embrapa.br/digital/bitstream/item/227322/1/CNPC-2021-Art-boletimCIM-16.pdf

Fernandes, A. A. O., Buchaman, D., \& Selaive Villarroel, A. B (2001). Avaliação dos Fatores Ambientais no Desenvolvimento Corporal de Cordeiros Deslanados da Raça Morada Nova. Revista Brasileira de Zootecnia, 30(5), 1460-1465.

Ferrel, C. L (1992). Nutrient requirements, other factors affect fetal growth. Feedstuffs, 17, 18-41.

Geron, L. J. V., Mexia, A. A., Garcia, J., Zeoula, L. M., Garcia, R. R. F., \& Moura, D. C. D (2012). Desempenho de cordeiros em terminação suplementados com caroço de algodão (Gossypium hirsutum 1.) e grão de milho moído (Zea mays 1.). Archives of Veterinary Science, 17(4), 34-42.

Machado, R., Simplicio, A. A, \& Barbieri, M. E (1999). Acasalamento entre ovelhas deslanadas e reprodutores especializados para corte: Desempenho produtivo até a desmama. Revista Brasileira Zootecnia, 28(4), 706-712.

Mohammadi, K., Beygi Nassari, M. T., Fayazi, J., \& Roshanfekr, H (2010). Investigation of environmental factors influence on pre-weaning growth traits in Zandi lambs. Journal of Animal and Veterinary Advances, 9(6), 1011-1014.

Muniz, E., Pires, C. C., \& Silva, J. H. S (1997) Crescimento ponderal e características de carcaça de cordeiros de diferentes genótipos. In: $34^{a}$ Reunião da Sociedade Brasileira de Zootecnia, Juiz de Fora. Anais..., Sociedade Brasileira de Zootecnia, 1:293-295.

Oliveira, D. C., Souza, G. N., Cordeiro, L. L., Carneiro, A. P. S., \& Carneiro, P. L. S (2010). Modelos de curvas de crescimento para ovinos cruzados. Revista da Estatística UFOP, 1, 1-2.

Rech, C. L. S., Rech, J. L., \& Fisher, V., et al (2008). Temperamento e comportamento materno-filial de ovinos das raças Corriedale e Ideal e sua relação com a sobrevivência dos cordeiros. Ciência Rural, 38(5), 1388-1393. 
Research, Society and Development, v. 11, n. 3, e17411326363, 2022

(CC BY 4.0) | ISSN 2525-3409 | DOI: http://dx.doi.org/10.33448/rsd-v11i3.26363

Rego Neto, A. A., Sarmento, J. L. R., Santos, N. P. S., Biagiotti, D. S. G. V., Sena, L. S., \& Guimaraes, F. F (2014). Efeitos ambientais sobre características reprodutivas em ovinos Santa Inês. Revista Brasileira de Saúde e Produção Animal, 15(1), 20-27.

Ribeiro, E. L. A., Koritiaki, N. A., Fernandes Junior, F., Constantin, C., \& Grandis, F. A (2013). Desempenho e rentabilidade: Desempenho e rentabilidade. Caderno Técnico e Cientifico: Revista Cabra e Ovelha, 41(79), 2-4.

Ribeiro, E. L. A., Silva, L. D. F., Mizubuti, I. Y., Rocha, M. A, Silva, A. P., Mori, R. M., Ferreira, D. O. L., \& Casimiro, T. R (2002). Desempenho produtivo de 70 ovelhas acasaladas no verão e no outono recebendo ou não suplementação alimentar durante o acasalamento. Semina: Ciências Agrárias, 23(1), 35-44.

Ribeiro, T. M. D., Monteiro, A. L. G., Prado, O. R., Natel, A. S., Salgado, J. A., Piazzeta, H. V. L., \& Fernandes, S. R (2009). Desempenho animal e características das carcaças de cordeiros em quatro sistemas de produção. Revista Brasileira de Saúde e Produção Animal, 10(2), 66-378, maio/jul.

Rocha, L., Fraga, A. B., \& Araújo Filho, J. T (2009). Desempenho de cordeiros cruzados em Alagoas, Brasil. Arquivo Brasileiro de Zootecnia, 221:145-148.

Sebrae (2020). Carne ovina é oportunidade de negócio para 2020. https://sebraers.com.br/ovinocultura/carne-ovina-e-oportunidade-de-negocio-para-2020/.

Silva, F. L. R., \& Araujo, A. M (2000). Características de reprodução e de crescimento de ovinos mestiços Santa Inês, no Ceará. Revista Brasileira de Zootecnia, 29(6), 712-1720.

Teixeira, M. C., Villaroel, A. B., Pereira, E. S., Oliveira, S. M. P., Albuquerque, I. A., \& Mizubuti, I. Y (2011). Curvas de crescimento de cordeiros oriundos de três sistemas de produção na Região Nordeste do Brasil. Ciências Agrárias, 33(5), 2011-2018.

Manzoni1, V. G.., Vaz, R. Z.., Ferreira, O. G., Costa, L. O., Denardin, A., \& Silveira, F. A (2017). Eficiência produtiva de ovelhas com diferentes carcaterísticas conformacionais sob pastejo. Ciência Animal Brasileira., 18(1-11), e 41123. 